\title{
FILOSOFÍAS ÉTICAS TRADICIONALES, SABIDURÍA POPULAR Y PERSONALIDAD
}

\author{
V. Pelechano \\ L. García \\ P. González-Leandro
}

Universidad de La Laguna (Tenerife)

\section{RESUMEN}

712 personas han cumplimentado una batería de pruebas de personalidad (modelos de los cinco grandes de Costa y McCrae) y cuatro pruebas de sabiduria. Los resultados indican que no existe una solución monofactorial a la sabiduria entendida como "saber de vida" y que las relaciones entre los cinco grandes y sabiduria son escasas, aunque se pueden detectar coeficientes estadisticamente significativos que tienen sentido psicológico. El papel de la edad(16-64 años) es pequeño y el de la intolerancia de la ambigüedad un tanto contrario a las expectativas. Los resultados se discuten en términos de niveles de consolidación de los factores de personalidad empleados tradicionalmente en las investigaciones asi como en el tipo de relaciones no lineales entre los dominios de personalidad y de sabiduria.

\section{Palabras clave: SABIDURIA COMO SABER DE VIDA, SABIDURIA $Y$ PERSONALIDAD.}

\footnotetext{
Trabajo presentado al 9th European Congress of Psychology, Granada, julio 2005

Este trabajo forma parte del proyecto BS-02002-00696 subvencionado por la Comisión Interministerial de Ciencia y Tecnología. Las opiniones y resultados vertidos en el trabajo son de exclusiva responsabilidad de los autores y en nada compromete a la entidad subvencionadora Correspondencia: vpelecha \&ull.es
} 


\section{SUMMARY}

712 Spanish adults (16-64 years old, both genders) have fulfilled the five-factor questionnaire by Costa and McCrae (NEO$P /-R)$ and four scales on wisdom as knowledge about important topics in life and in personal relationships. The results show: (a) the wisdom scales give a multifactorial solution; (b) the role of personality dimensions is scarce in correlation analysis; (c) age plays little role in the relationship among personality and wisdom criteria; $(d)$ intolerance of ambiguity pays a role very different to expectations proposed by the Berlin group and $(e)$ relationships among personality and wisdom can be found in non-linear models and in a different conceptualization of personality variables (more restricted in amplitude and referred to contextual world of life).

Key words: WISDOM AS RELEVANT KNOWLEDGE ABOUTLIFE PROBLEMS, PERSONALITY AND WISDOM.

\section{PSICOLOGÍA Y SABIDURÍA}

El desarrollo histórico de la psicología, en general, ha transcurrido por unos caminos que han estado alejados del campo de "la sabiduría" y en nuestros días, la "psicología de la sabiduría" resulta una expresión no sólo poco frecuente sino también activadora y/o generadora de actitudes de extrañeza y sorpresa incluso en el mundo académico. Las razones son muy complejas aunque fundamentalmente, la psicología se alejó de la sabiduría en la medida en que ésta desprendía un cierto tufillo a filosofía y, por lo mismo, incontrastable y tan variada como número de pensadores ha habido (Pelechano, García y Fumero, 2004). Su reinserción está siendo capitaneada por psicólogos evolutivos (desde consideraciones de ciclo vital, pasando por la evolución de la fase post-formal en el desarrollo cognitivo, el estudio del envejecimiento humano) y por clínicos y personólogos (psicología positiva, ampliación del universo de pensamiento que ha estado cubierto por concepciones monolíticas de inteligencia que diluían las competencias humanas relevantes para la supervivencia e incluso la felicidad en una "inteligencia general humana". Con unos antecedentes hacia los años 
treinta del pasado siglo, realmente la psicología de la sabiduría arranca en los años 80 y se va consolidando en unas líneas de trabajo, la más abundante y rica posiblemente sea la que corresponde al grupo de Baltes, actualmente en Berlín.

El presente trabajo no entra en polémica con dicho grupo y no se plantea, como se ha hecho recientemente (Ardelt, 2004; Baltes y Kunzmann, 2004), la polémica respecto a lo que sea y no sea sabiduría (si es tan solo un aspecto cognitivo o algo más que ello). El autor senior de este trabajo está presentando en los últimos años una manera de pensar y hacer en la psicología de la sabiduría que resulta un tanto distinta a la del grupo de Baltes y a la de Monika Ardelt. Entre las líneas básicas del discurso de este replanteamiento se encuentran las siguientes:

(a). La sabiduría representa una excelencia humana que compromete cierto tipo de cognición no reducible a la evaluada por los tests de inteligencia convencionales y tampoco por la cognición social, tal y como se expone en los trabajos publicados al uso, ni por la "inteligencia práctica" tal y como la entiende Sternberg. Compromete, al menos, ciertos procesos cognitivos, la emocionalidad y cuestiones éticas básicas, cuya delimitación es muy compleja y no bien conocida, ni menos resuelta, en nuestros días.

(b). Las cogniciones que forman la sabiduría no se reducen al mundo de las relaciones interpersonales, sino que incluyen al mundo profesional, al conocimiento de los elementos sustantivos de la realidad $y$, al menos, un tipo de operaciones cognitivas que se han llegado a denominar como "reflexividad", para referirse al hecho de que el primer y más claro significado de las expresiones esconde una segunda y hasta una tercera significación, a la que se llega tras una reflexión y trascendencia de este primer nivel. Un ejemplo se encuentra en el mundo de los refranes y así, por ejemplo, "El aceitero las manos se unta" se está refiriendo tanto al "aceite" como a todo aquello que pasa por las manos o la decisión del ser humano; se refiere tanto a los que cometen fraudes económicos, políticos (poder), como a los que se manejan cuestiones de confianza. Este añadido de significación convierte el conocimiento sabio en una suerte de hermenéutica que sigue unas reglas bastante precisas (aunque tampoco bien conocidas) y a cuyo dominio no llegan todos los seres humanos en su evolución 
"natural-social". Y ello implica, además, que un plano de análisis estrictamente funcional-inmediato o a corto plazo resulta poco adecuado para el estudio de la sabiduría, porque elimina, precisamente, ese plus significativo.

(c). Una de las pretensiones de la sabiduría es que se refiere a un tipo de conocimiento "intemporal" en el sentido de que su valor trasciende al contexto espacio-temporal en el que se ha formulado por primera vez. De ahí que existan "depósitos" de sabiduría en ciertos libros como la Biblia o el Tao; en el mundo de los proverbios, refranes $y$ decires que se conservan en la mentalidad y cultura popular $y_{\text {, }}$ asimismo, en la obra de grandes pensadores a lo largo de la historia. Es en este contexto en el que se ubica el presente trabajo que trata de entresacar correlatos de dos filosofías de vida propuestas por primera vez en occidente en la época helenística (epicureismo y estoicismo). Se incorpora asimismo un factor de sabiduria popular encarnado en refranes españoles y que hemos calificado como "prudencia-astucia interpersonal".

(d). En las distintas revisiones de la bibliografía sobre sabiduria tiende a encontrarse una referencia (en la mayoría de ocasiones se incorpora como componente) a la tolerancia de la incertidumbre o de la ambigüedad. Se ha dicho en repetidas ocasiones que la persona sabia debe tolerar la incertidumbre sin tomar partido inmediato sobre una u otra opción que se presente y en el grupo de Baltes, la tolerancia de la incertidumbre constituye uno de los cinco criterios utilizados para definir la sabiduría como un sistema experto sobre las cuestiones de vida (si bien es un criterio "secundario"). Desde la posición que se defiende en el modelo que proponemos, el papel de la tolerancia o intolerancia de la incertidumbre o de la ambigüedad es más complejo, en la medida en que una respuesta "sabia" no siempre lleva consigo ser tolerante con la ambigüedad: la situación o el problema puede ser entendido como ambiguo por una persona en la medida en que no posea el volumen de conocimiento suficiente $y$, por lo mismo, el sabio que tenga ese volumen de conocimientos, puede ser escasamente tolerante con la ambigüedad en estas situaciones (si posee el conocimiento adecuado para la solución del problema). Debería diferenciarse entre la falta de control impulsivo y la intolerancia de la ambigüedad en la medida en que una cosa es actuar sin pensar y, en 
la gran mayoria de los casos, de manera inconsciente y errática y otra cosa es ofrecer la respuesta precisa, adecuada, rápida y altamente satisfactoria que puede requerir la reconducción de una situación o la solución de un conflicto que posee aristas y protagonistas muy claros..., al menos para "el sabio" . Las predicciones respecto a la relación y papel de la ambigüedad en la sabiduria son complejas y dependen de situaciones, problemas y momentos de vida. $Y$, por otra parte, una cosa es la sabiduría y otra harto distinta, identificar esa "filosofía" de distanciamiento de la realidad con ella, frente al pensamiento romántico, intuitivo y rápido que puede asimismo, ser "sabio".

Por lo que se refiere a la intolerancia de la ambigüedad, en suma, no necesariamente formaría parte de las filosoffas de vida / o sabidurías de vida, sino que sería un elemento complementario que desempeñaría un papel en ciertos contextos de significación, pero no necesariamente en todos.

(e). Al igual que sucede con otros criterios de "excelencia" (como la genialidad), la sabiduría no debería entenderse como un rasgo básico del funcionamiento personal que se manifiesta en todos los contextos de vida y en todas las situaciones vitales. El "sabio" no está obligado a que todas sus obras sean identificadas como "sabias" y excelentes ${ }^{2}$. Bastaría con unos actos de excelencia para que una persona pueda ser calificada como sabia, aunque en la actualidad no está claro cuántos actos sabios son necesarios y, desde ahí, el criterio de corte que diferenciaría a la persona sabia de la que no lo es. Como sugerencia se apunta que aunque no todos los actos tengan necesariamente que ser "sabios", al menos, la tasa basal media de sus acciones se encuentre por encima de la de sus conciudadanos, al menos en lo que respecta a "sabiduria".

\footnotetext{
1 Recuérdese al respecto el refrán prescriptivo "El pasmo se ataja antes de que entre", - la promoción de prevención. Aunque ello no implica una justificación "sabia" de la "guerra preventiva", obviamente.

"Salomón, después de su "juicio" parece que hizo bastantes acciones no solamente poco sabias, sino anti-sabias y poco recomendables (por ejemplo, renegar del dios de sus padres y unirse a la reina de Saba, que le llevó a alejarse de sus comienzos...). Al final, sin embargo, retornó al buen camino "sabio" tras separarse de esa "mujer gentil y contraria a la fe del dios de sus padres". Otra consideración se refiere a que no existe un critenio claro respecto a cuántas acciones sabias se requieren para que una persona sea calificada como "sabia".
} 
(f). El conocimiento que lleva a la sabiduría trae consigo unos concomitantes emocionales (de igual valor que el dominio cognitivo) que, sin pretensión de agotar su número podría contabilizar, al menos, el autocontrol personal, la cordialidad, la minuciosidad (sin perder la idea central), el sentido de la coherencia, la fortaleza personal y cierta introversión. Sin embargo, la manera de evaluar estas cualidades debería ser con instrumentos que se movieran en un rango intermedio de generalidad y que no se limitasen tan solo a cuestionarios autoaplicados de personalidad y pretendidamente referidos a aspectos básicos genéricos del funcionamiento personal descontextuados.

(g). Alternativamente a la concepción categorial (ser o no ser sabio un ser humano, un pensamiento o una acción) se propone que cada una de las acepciones de "sabiduría" es susceptible de ser conceptualizada como un conjunto de atributos cuantitativos que se encontrarían presentes en todos o la mayoría de ciudadanos, aunque en intensidad o cantidad distinta (todos "participaríamos" de las características psicológicas que dan lugar a acciones y decisiones sabias, aunque la cuantía o la estructura fuera distinta en unas y otras personas). En el modelo general de parámetros que defiende el autor senior de este trabajo (Pelechano, 1997, 2000, Pelechano y Servando, 2004), las relaciones que cabe esperar entre los distintos atributos no son constantes ni necesariamente lineales y por ello, en función de los indicadores utilizados de "emocionalidad" y de "sabiduría", así como de las puntuaciones alcanzadas en ellos, las relaciones pueden variar en intensidad, e incluso en sentido (positivo o negativo).

(h) No existe solamente una acepción de sabiduría. Deberían diferenciarse, al menos, las siguientes: conocimientos acerca de la estructura última de la realidad física, social y espiritual del ser humano y su mundo; conocimientos y acciones referidas a "saber vivir" y/o vivir de manera que se sea lo más feliz posible con medios no intrusitos con los demás (o sabiduría de vida); sabiduría como cúmulo de conocimientos útiles en el mundo interpersonal y social que pueden tener una aplicación profesional (muy cercano a la idea de experto, si bien en grado extremo) en el mundo contemporáneo y, finalmente, la sabiduría de corte religioso-espiritual en la que se identifica con el conocimiento de dios o de la relación ser humano-dios. 
En la actualidad no existen procedimientos claros de conversión de unas acepciones a otras, ni se encuentran todas las acepciones desarrolladas dentro del camino de la ciencia, ni son todas ellas susceptibles de análisis científico. Las que se han intentado estudiar, o se han operacionalizado con mayor éxito son las que se encuentran directamente relacionadas con el "saber vivir" y la "buena vida", en sus dimensiones interpersonales.

Estas acepciones se encuentran muy influidas por variables de tipo cultural $y$, asimismo, se encontrarian diversificadas dentro de una misma cultura por las formas distintas de entender la "buena vida", asi como los mejores procedimientos para obtenerla. La complejidad de la sociedad contemporánea sugiere que pueden existir distintos nichos culturales que, si bien pueden tener relaciones entre sí, nichos que conforman modos de ver la realidad de forma distinta y con una forma distinta de vivir esa realidad y de lograr la "felicidad" que se supone acompaña a esa "buena vida". De ahí una de las ideas matrices de los trabajos que hemos publicado: que existen distintas formas de entender y operacionalizar la sabiduría, incluso dentro de esa "subdivisión" que la asimila a saber vivir.

(i). Las plasmaciones de conocimientos y acciones sabias se encuentran recogidos, entre otros, en libros y tradiciones orales. En este trabajo se presentan datos sobre dos instrumentos inspirados en dos filosofías de vida con una fuerte impronta ética, que aparecieron en occidente durante la época helenística y que han tenido una pervivencia desigual aunque en la actualidad se encuentran presentes en el pensamiento contemporáneo: el estoicismo y el epicureismo. Junto a ello, la sabiduría popular o lega (refranes) y la intolerancia a la ambigüedad.

En la medida en que los procesos psicológicos comprometidos con la sabiduría, en cualesquiera de las acepciones antes mencionadas, son procesos muy complejos, se deberia procurar, en primer lugar, aislar variables que fueran teóricamente relevantes y, por otro lado, anclar estas variables en marcos teóricos o dominios conocidos de funcionamiento psicológico, con el fin de poder apresar tanto la comunidad con estos dominios, como la especificidad de este campo de estudio.

Las dos orientaciones filosóficas sustratuales a las pruebas que se han elaborado (estoicismo y epicureismo) poseen implicaciones 
indudables acerca del desarrollo humano y el funcionamiento moral, lo que no ha sido del agrado del desarrollo de la psicología científica en general (Baltes, Glück y Kunzmann, 2005) al encontrarse, de lleno, cargadas de juicios de valor y prescripciones acerca de la naturaleza humana y su logro de felicidad. $Y$ algo similar, por lo que se refiere a contenidos valorativos, sucede con la prueba de refranes elaborada sobre prudencia-astucia que funcionaría como marcadora de concepciones legas de sabiduría. El componente sobre intolerancia de la ambigüedad se trata más adelante.

El análisis lógico de estos dos acercamientos (estoicismo y epicureismo) ha demostrado que presentan ciertas incoherencias y hasta contradicciones, que su insistencia en una posición individualista y hasta de "alejamiento" de los demás, un clasismo intelectual y personal y el desgajamiento del mundo físico y social representan elementos que no parecen estar adaptados al entorno cultural en el que nos encontramos, aunque algunos de sus elementos principales parecen seguir estando vigentes. Con el fin de evitar propuestas dicotómicas radicales, en uno de los instrumentos se ha utilizado la técnica de ofrecer dos formulaciones en cada ítem y pedir a los participantes que elijan una de ellas (una de ellas es coherente con la posición filosófica y otra no, aunque no necesariamente es contradictoria con respecto a ella). De las dos opciones filosóficas, una de ellas terminó su evolución con la muerte del fundador (Epicuro) mientras que el estoicismo fue evolucionando y muchos de sus componentes se incorporaron a la moral cristiana y se encuentran presentes en nuestros días. En este sentido, una de las ideas es ofrecer información acerca de la "presencia" de estos dos acercamientos ético-comportamentales que fueron modelos de "sabiduría" en su época y que parecen haber sobrevivido al paso del tiempo. Admitiendo, de entrada, su valor restrictivo, y su falta de coherencia interna. En otros instrumentos se incorporan otras maneras de pensar y que llevarían a prácticas distintas (por ejemplo, la dimensión aislada por la prueba de refranes es harto distinta de las que cubren estoicismo y epicureismo. 


\section{MÉTODO}

\section{Participantes}

En total han participado 711 adultos de ambos sexos (68\% mujeres); la mayoria $(73 \%)$, estudiantes universitarios de psicología de la Universidad de La Laguna. La media de edad ha sido de 26,35 años con un rango entre 16 y 64 años. Los estudiantes corresponden a tres promociones distintas de la Facultad de Psicología. Por lo que se refiere al resto de muestra se encuentra formada por estudiantes de bachillerato (secundaria no obligatoria) y adultos conocidos de los estudiantes universitarios que han colaborado voluntariamente en la investigación (técnica de "bola de nieve") y estos adultos cubren un amplio rango de niveles profesionales, aunque su distribución no es normal.

\section{Instrumentos}

Los participantes han cumplimentado dos tipos de pruebas: cuestionarios de personalidad y pruebas de sabiduria.

Por lo que se refiere a cuestionarios de personalidad, en este trabajo nos restringimos al cuestionario NEO-PI de Costa y McCrae (1992), adaptado a la población española (Avia, Sanz y Bernardos, 1997) y corregido en algunos de los ítems con el fin de adecuarlos a la significación original de los autores (de Miguel y Pelechano, 2000). Aísla cinco grandes factores básicos de personalidad: neuroticismo, extraversión, apertura a la experiencia, cordialidad y escrupulosidad, cada uno de ellos con seis facetas que componen un amplio rango semántico en la significación de los factores. Se trata del último gran cuestionario difundido en muchos países y sobre el que existe un considerable volumen de investigación. Neuroticismo y extraversión guardan relaciones estrechas con afectividad negativa y positiva respectivamente, el primero relacionado además con inestabilidad emocional y el segundo con sociabilidad. Los índices de consistencia interna son altos, por encima de 0,80 en todos los factores. Los factores de apertura a la experiencia y cordialidad son claramente factores "positivos"; el último factor, al que se ha denominado tentativamente 
escrupulosidad (conscientiousness) recoge elementos de preocupación por el cumplimiento del deber, tareas bien hechas y cuidado en la realización de las mismas, que, en función de los contextos de medida puede ser considerado como un elemento facilitador o perturbador de la integración personal.

Por lo que se refiere a los factores de sabiduría se han utilizado cuatro cuestionarios, todos ellos previamente estudiados en cuanto a composición factorial y validez interna y diferencial:

(a). Cuestionario de pensarniento estoico de Pelechano (García y Pelechano, 2004), elaborado a partir de las versiones de Séneca y Epicteto. Originalmente formado por 35 elementos del tipo "Pensar en la muerte y en el sufrimiento que lleva consigo no tiene sentido: cuando se está muerto no se siente nada", o "Si amo a alguien, el problema lo tengo yo porque amo, y no la persona amada". Existen 4 alternativas de respuesta en función de la probabilidad que se atribuya a poner la afirmación en práctica (de nunca a siempre, en todas las ocasiones) y se han formulado elementos que van en la línea coherente con el estoicismo y otros que van en contra de él. El análisis factorial arrojó una solución factorial de tres factores en primer orden (oblimin) y que dio lugar a un factor de segundo orden robusto que recoge la variabilidad empírica y muestra ciertas incoherencias en el sentido de que parecen "convivir" bien en nuestros días enfoques estoicos y antiestoicos, sin conciencia de contradicción (al menos sin conciencia de diferencias ente ellos y formando parte de un mismo sistema de valores).

(b). Cuestionario de pensamiento epicúreo de Pelechano (Pelechano, en prensa). Construido a partir de la obra Máximas capitales de Epicuro y a sabiendas de que se trata de un acercamiento que presenta algunas incoherencias, la prueba original consta de 22 pares de afirmaciones. La tarea consiste en leer cada par y elegir una de ellas como la preferida a la hora de poner una de las dos en práctica. Cubren un amplio espectro de campos que van desde la primacía del conocimiento sobre la acción (por ejemplo: "Necesitamos conocernos a nosotros mismos, a los demás y a la naturaleza para poder elegir qué cosas debemos y qué cosas no debemos hacer. De este modo haremos las cosas que producen más felicidad y tienen menos efectos malos para nosotros" frente a "Lo que necesito es pasármelo bien, 
porque todo aquello que hace que me lo pase bien, es bueno para mi") hasta prescripciones respecto a relacionarse con los demás y tener confianza en ellos (como "Uno de los mayores placeres es tener la confianza de los que nos rodean y confiar en ellos. Así sabemos qué hacer y cómo hacerlo", frente a "Quienes tienen la confianza de los que les rodean y confian en ellos son los primeros candidatos a sufrir (porque les fallen los demás, porque se mueren, etc.)". Se han aislado emplricamente tres factores de tercer orden (a partir de componentes principales y rotación oblimin) que no son siempre coherentes con la filosofía epicúrea: control/independencia personal con jerarquía social y acepción negativa de placer; inmanencia y dicotomía excluyente placer/dolor y devaluación de dolor físico ${ }^{3}$. Se ha utilizado asimismo una escala criterial de epicureismo, a partir de las "puntuaciones" positivas en el sentido epicúreo.

(c). Cuestionario de refranes de Pelechano (Pelechano, Fumero y Garcia, 2004). Se trata de un cuestionario formado definitivamente por 48 refranes referidos a las relaciones interpersonales y que ha dado una solución monofactorial. Ejemplos de ítems son los siguientes: "Lo que se calla, se puede decir; lo que se dice, no se puede callar", "Cumple con todos, fía de pocos", "Lo que no se puede remediar, hay que aguantar", con 4 alternativas de respuesta respecto al grado de frecuencia de puesta en práctica del refrán en la vida cotidiana. El análisis factorial dio lugar a un factor de segundo orden robusto que se denominó tentativamente como prudencia-astucia en relaciones personales y que contiene una prudencia en el hacer y decir con los demás y, a la vez, no acabarse de fiar de ellos y "ver un poco más allá" de las situaciones concretas de interacción.

(d). Cuestionario de intolerancia de la ambigüedad para adultos, de Pelechano, presentado en Pelechano y Aguilera (2004) en cuanto a consistencia interna, validez factorial, validez diferencial y convergente con grupos criterio y otras variables de personalidad. Está formado

\footnotetext{
${ }^{3}$ El incremento en volumen de muestra ha aconsejado una nueva factorización respecto a la presentada en Garcia y Pelechano (2004), que ha sido presentada en Pelechano (en prensa) y es la que se utiliza aquí. En esta nueva versión se ha ganado en consistencia interna (oscila entre 0,50 y 0.79 ). El empleo de la escala criterial (formada por la suma de los tres factores en el sentido epicúreo) se ha empleado en aras de un posible incremento en poder predictivo respecto a los factores de personalidad.
} 
por ítems referidos fundamentalmente a situaciones y decisiones en contextos de vida interpersonal, aunque se ofrecen cuestiones generales con el fin de estudiar si se trata de un factor básico-general o deben aislarse componentes diversos. Ejemplos de ítems son los siguientes: "Todo aquello que no puede formularse en términos simples, o es malo, o no sirve para nada, o está mal planteado", "La duda ante los problemas forma parte de la debilidad" o "Quien tarda mucho en decidir qué hacer no es sabio, es inseguro". Se elige, para cada cuestión, si es verdad o mentira. Está formado originalmente por 64 elementos y dio una solución monofactorial en segundo orden.

\section{Procedimlento recogida de información}

Como información complementaria a la expuesta en la descripción de la muestra hay que señalar que la correspondiente a universitarios se logró a base de estudiantes de la materia de psicología de la personalidad, que desearon participar en el estudio durante tres promociones y recibían un ligero incremento de nota por ello, si aprobaban la materia por evaluaciones. Algunos de estos alumnos, además, participaron en una estrategia de bola de nieve para recoger información de sus familiares y amigos (se les daban las pruebas dentro de un sobre y las personas colaboradoras mandaban este sobre cerrado ya franqueado al Departamento de Personalidad, Evaluación y Tratamientos Psicológicos de la Universidad de La Laguna). Aquellos participantes que así lo expresaron, al final de cada curso podian recoger los resultados que habían alcanzado en las pruebas mediante entrevista con los profesores de la materia y después de la correspondiente identificación. En todos los casos se mantuvo la privacidad de las respuestas. Las respuestas dadas a los cuestionarios se transformaron en puntuaciones y los cálculos fueron hechos en PC con el paquete SPSS-12.

\section{HIPÓTESIS RACIONALES}

1. La existencia de más de una forma de sabiduría llevaría consigo la obtención de una estructura multifactorial si se lleva a cabo un análisis factorial de todas las pruebas de sabiduría que se han utilizado. 
Repárese que todas las pruebas son de papel y lápiz y, por ello, tendrían en común una parte de la "varianza del método" y si, aun con el mismo tipo de instrumento y cubriendo dominios semánticos distintos se obtiene una estructura multifactorial, ello sería indicador de la existencia de más de una manera de entender la sabiduría, con plasmaciones independientes entre sí y que exigirían maneras de validación y de entrenamiento distintas.

2. Aunque la "sabiduria" forma parte del funcionamiento personal, las relaciones con las dimensiones de personalidad deben ser escasas (resultados con pruebas distintas que se han obtenido en otras ocasiones y en especial por el grupo de Baltes en Berlín). En líneas generales deberían esperarse relaciones negativas con neuroticismo (o afectividad negativa) y escrupulosidad; nada relevantes con extraversión social y positivas aunque muy tenues con cordialidad y apertura a la experiencia en la medida en que las "dimensiones positivas" de la estructura personal tienden a ser entendidas como positivas en contextos de socialización "agradable", mientras que los factores de sabiduría elegidos tienden a ser escasamente promotores de la interacción social y más bien representan un cierto distanciamiento de esa interacción o, al menos, tienden a representar una cierta desconfianza en toda interacción social que no sea con "sabios" (lo que, desgraciadamente, no suele abundar, por la escasez de sabios). En todo caso, esta independencia o escasa relación sería una prueba de que no debería identificarse sabiduría con socialización positiva ni "bienestar" identificado como afectividad positiva, como tiende a encontrarse recogido en los autores que trabajan en temas tales como bienestar personal y felicidad (Fredrickson, 2001, 2005; Fredrickson y Losada, 2005; Argyle, 2003).

\section{RESULTADOS}

\section{La estructura factorial de las escalas de sabiduria}

Un volumen de 421 participantes cumplimentaron los instrumentos de sabiduría. Las escalas de estoicismo y epicureismo han sido factorizadas y en el caso del estoicismo se ha encontrado una solución monofactorial de segundo orden. En el caso del epicureismo la solución 
de tercer orden ha sido de tres factores, aunque el número de elementos en cada uno de estos elementos es pequeño, con lo que la consistencia interna es relativamente baja. De ahí que se haya elegido la conformación de una puntuación única de epicureismo formada por la suma de los tres factores "coherentes" con la clave epicúrea de corrección (en algún caso). Con el fin de conocer el papel de la edad se ha incluido asimismo, como variable. Se ha pedido una rotación varimax a partir de componentes principales. La matriz factorial rotada, en la que se incluyen solamente las variables con un peso factorial igual o mayor de 0,40 se encuentra en la tabla 1.

Tabla 1. Solución factorial rotada $(\mathrm{N}=421)$ de las variables de sabiduria empleadas en este estudio. La explicación en el texto

\begin{tabular}{|l|c|c|c|c|c|}
\hline VARIABLES & F-I & F-II & F-III & F-IV & COMUNAL \\
\hline Estoicismo & 0,85 & & & & 0,74 \\
\hline $\begin{array}{l}\text { Intolerancia } \\
\text { ambigüedad }\end{array}$ & 0,81 & & & & 0,70 \\
\hline $\begin{array}{l}\text { Prudencia / } \\
\text { astucia } \\
\text { interpersonal }\end{array}$ & & & 0,99 & & 0,99 \\
\hline Epicureismo & & & & 0,99 & 0,99 \\
\hline Edad & & 0,99 & & & 0,98 \\
\hline
\end{tabular}

Nota.- F-I, F-II, F-III y F-IV factores definidos por las saturaciones $>0,40$ en la solución factorial; COMUNAL=Comunalidad.

La solución se presenta como muy clara y apenas necesita comentarios:

1. No existe una solución monofactorial de las escalas de sabiduría utilizadas. Para explicar la mayor parte de la varianza total $(79,15 \%)$ se requieren cuatro factores, de los que tres se refieren a escalas de sabiduría.

2. La edad no parece desempeñar un papel relevante (recuérdese que se trata de una muestra entre 16 y 64 años) en la sabiduría evaluada por estas pruebas.

3. El primer factor está representado por las escalas de estoicismo y de intolerancia de la ambigüedad: las dos saturaciones están por encima de 0,80 . Este resultado sugeriría que la sabiduría estoica es la única relacionada con la ambigũedad y que la relación sería positiva, 
de mayor "sabiduría" a mayor intolerancia de la ambigüedad: el más estoico no es mas ambiguo, sino todo lo contrario.

4. Y, por otro lado, la intolerancia de la ambigüedad es independiente del epicureismo y de la prudencia-astucia interpersonal evaluada por la escala de refranes.

\section{Dimensiones de personalidad y criterios de sabiduría}

Los resltados correspondientes a correlaciones Pearson se encuentran recogidos en la tabla 2 , con expresión del nivel de significación estadistica de los coeficientes (se han omitido los que no han dado un nivel de significación, $\mathrm{N}=286$ ).

Tabla 2. Coeficientes de correlación estadísticamente significativos entre escalas de sabiduria y dimensiones de personalidad (cincos grandes). La explicación en el texto

\begin{tabular}{|l|l|l|l|l|l|}
\hline VARIABLES & Neurotic & Extrav & Apert & Cordial & Escrupul \\
\hline Estoicismo & $-0,30^{\star *}$ & & & $0,35^{\star *}$ & $0,23^{\star}$ \\
\hline Epic1 & & & $0,10^{*}$ & $0,21^{\star}$ & $0,12^{\star}$ \\
\hline Epic2 & & & & & $-0,15^{\star}$ \\
\hline Epic3 & & & $0,18^{*}$ & & \\
\hline Astprudenc & $0,19^{\star}$ & & & & \\
\hline
\end{tabular}

Nota. - Neurotic $=$ neuroticismo; Extrav $=$ extraversión; Apert $=$ apertura a la experiencia; Cordial = Cordialidad; Escrupul = escrupulosidad; Estoicismo = factor general de estoicismo (constructivismo mentalista y restricción de placeres a lo controlable); Epic1 = Control/independencia personal con aceptación de jerarquía y acepción negativa de placer (placer como ausencia de dolor); Epic2 = Inmanencia vida (negación valor de muerte y vida después de la vida); Epic3 = Dicotomía placer/ dolor y devaluación dolor físico (si es muy intenso, se pierde conciencia).

De entrada hay que decir que los coeficientes que alcanzan el nivel de significación estadística no son muchos y no son de cuantía alta. EI mayor porcentaje de varianza común entre escalas de sabiduría y dimensiones de personalidad no pasa del $10 \%$, por lo que cabe afirmar que las escalas de sabiduría no son identificables con alguna de las cinco "grandes" o importantes dimensiones del modelo de Costa y McCrae.

De todas las variables es el factor de segundo orden de estoicismo el que presenta mayor volumen de relación. Este factor abarca la idea 
de que la mayor fuerza para el logro del bienestar personal y la sabiduría es construirse un mundo en el que no se desee más que aquello que se encuentra a nuestro alcance, un cierto clasismo en las relaciones personales y no estar pendiente de los demás para la toma de las decisiones propias e importantes. Se relacina significativa y negativamente con neuroticismo $(-0,30, p<0,01)$ y positivamente con cordialidad $(0,35, p<0,01)$ y escrupulosidad $(0,23, p<0,05)$.

El primer factor de epicureismo (control/independencia personal con aceptación de jerarquía y de una acepción negativa del placer) presenta relaciones con tres factores "positivos" de personalidad: apertura intelectual a la experiencia $(0,10, p<0,05)$, cordialidad $(0,21, p<0,05)$ y escrupulosidad $(0,12, p<0,05)$.

El segundo factor de epicureismo (inmanencia de vida, con negación de la muerte como fenómeno psicológico y7o de sufrimiento) presenta solamente un coeficiente estadísticamente significativo negativo y con escrupulosidad $(0,15, p<0,05)$ indicando cierta tendencia a despreocupación por tareas minuciosas y bien hechas, si bien el coeficiente no es relevante.

El tercer factor de epicureismo (dicotomía excluyente de placer/ dolor con devaluación del dolor físico) se relaciona apenas positivamente con la cordialidad $(0,18, p<0,05)$.

Finalmente, el factor de astucia/prudencia de la prueba de los refranes presenta dos coeficientes significativos estadisticamente: 0,19 $(p<0,05)$ con neuroticismo y $-0,16(p<0,05)$ con escrupulosidad, indicando que la inestabilidad emocional-afectividad "negativa" tenderia a promover ligeramente esta prudencia y tomar distancia en las relaciones sociales; y por otro lado, que esta astucia/prudencia dificulta un análisis y aceptación fina de deberes sociales y la insistencia en los detalles que llevan a una tarea bien hecha.

Con el fin de complementar los análisis correlacionales, se llevaron a cabo ecuaciones de regresión en los que la variable dependiente era cada uno de los factores de sabiduría independientemente. Solamente el factor de estoicismo presentó un coeficiente estadísticamente significativo con una ecuación formada por más de una variable: neuroticismo (beta tipificada de $-0,292, p<0,0006$ ) y cordialidad (beta tipificada de $0,199, p<0,005)$. En los tres factores de epicureismo asimismo se encontraron coeficientes beta estadísticamente 
significativos, una variable para cada factor: cordialidad (epi1), escrupulosidad (epi2) y neuroticismo (epi3).

Es posible que las relaciones se encuentren en unas puntuaciones y no en otras (relaciones no necesariamente lineales) y, para acercarnos a esta posibilidad se llevó a cabo una división de la muestra en puntuaciones extremas para cada factor de sabiduría (criterio de $20 \%$ superior e inferior como grupos extremos). Los resultados correspondientes a diferencias estadísticamente significativas se encuentran en la tabla 2, con expresión del número de participantes en cada grupo y variable de contraste, medias, desviaciones típicas de cada subgrupo y valor de " $t$ " de la diferencia de medias. Con el fin de ofrecer una imagen más clara al lector, en las figuras $1,2,3,4$ y 5 se encuentran representadas, gráficamente, estas diferencias estadisticamente significativas.

Tabla 3. Tabla resumen de diferencias estadísticamente significativas entre grupos extremos (20\% superior e inferior) de puntuaciones en cada factor de sabiduria. Medias correspondientes a puntuaciones en personalidad. La explicación en el texto

\begin{tabular}{|l|l|l|l|l|l|l|l|r|}
\hline VARIAB. & PERS & $\mathrm{N}$ & $\mathrm{M}$ & $\mathrm{DT}$ & $\mathrm{N}$ & $\mathrm{M}$ & $\mathrm{DT}$ & $\mathrm{T}$ \\
\hline F1epic & Cordialidad & 155 & 116,85 & 16,08 & 206 & 121,51 & 15,43 & $-2,78$ \\
& Escrupul. & 153 & 110,68 & 21,41 & 208 & 115,86 & 18,55 & $-2,46$ \\
\hline F2epic & Ast/Prud & 140 & 81,75 & 13,23 & 181 & 80,22 & 15,24 & $-2,20$ \\
\hline F3epic & Neurot & 116 & 93,62 & 20 & 96 & 87,88 & 21,24 & 1,99 \\
& Apertura & 132 & 113,07 & 16,53 & 91 & 121,06 & 16,75 & $-3,70$ \\
\hline Estoic. & Neurot & 83 & 101 & 20,95 & 71 & 85,25 & 17,70 & 5,06 \\
& Extrav. & $\mathbf{8 3}$ & 108,16 & 18,53 & 65 & 116,25 & 16,82 & $-2,84$ \\
& Cordial. & 93 & 111,86 & 16,03 & 70 & 125,40 & 13,40 & $-5,72$ \\
& Escrupul. & 88 & 107,20 & 20,20 & 70 & 120,24 & 17,39 & $-4,28$ \\
\hline Astucia/ & Neurot. & 94 & 85,78 & 21,02 & 79 & 96,23 & 21,15 & $-3,25$ \\
prudencia & Escrupul. & 102 & 119,32 & 17,96 & 84 & 111,06 & 22,15 & 2,75 \\
\hline
\end{tabular}

Nota.- El número de participantes en cada subgrupo es distinto en la medida en que existe un número distinto de personas que cumplen el criterio de agrupación (20\% superior e inferior). El nivel de significación de " 4 " es $p<0,05=1,96 ; p<0,01=$ 2,$58 ; p<0,001=3,43$. $N=$ número de personas quew forman cada grupo; $M=$ media en factor; $\mathrm{DT}=$ desviación tipica en factor.

F1epic $=$ Control/independencia personal con jerarqula y acepción negativa de placer; F2epic = Inmanencia (devaluación de muerte y de vida después de la vida); F3epic = Dicotomia placer/dolor y devaluación de dolor f́sico. 
Figura 1. Diferencias estadisticamente significativas de personalidad en grupos extremos de punturaciones en factor primero de epicureismo. La explicación en el texto

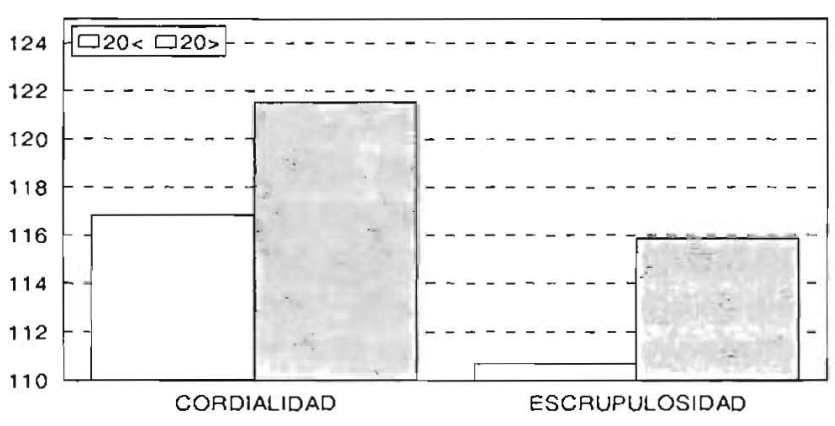

Figura 2. Diferencia estadísticamente significativa de personalidad en grupos extremos (20\%) de segundo factor epicureismo en astucia/prudencia con refranes. La explicación en el texto.

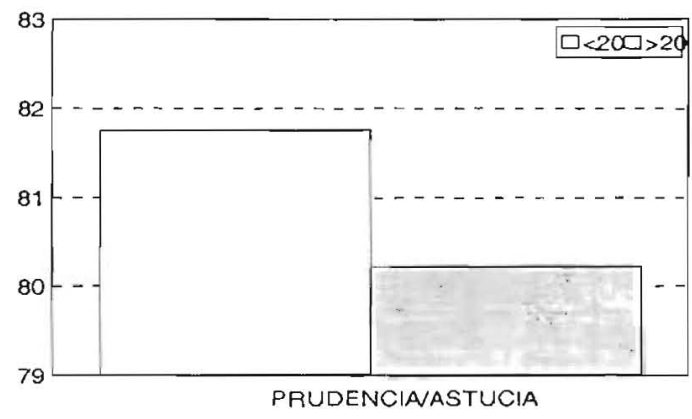

Figura 3. Diferencias estadísticamente significativas de personalidad en grupos extemos (20\%) de factor tres de epicureismo. La explicación en el texto

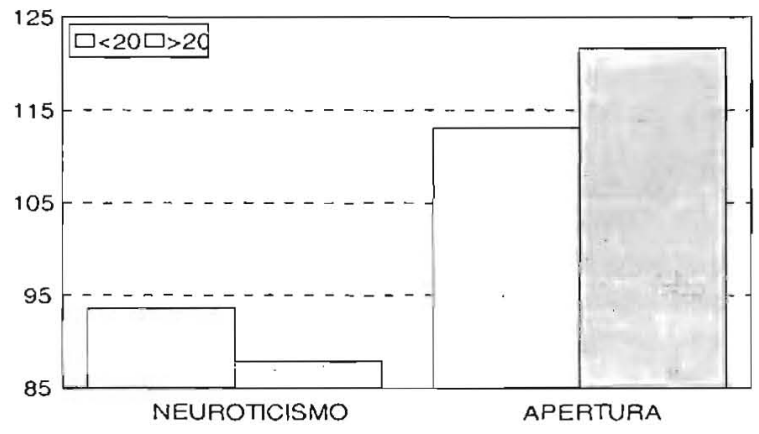


Figura 4. Diferencias estadisticamente significativas en medias de personalidad para grupos extremos (20\%) de sabiduría estoica. La explicación en el texto

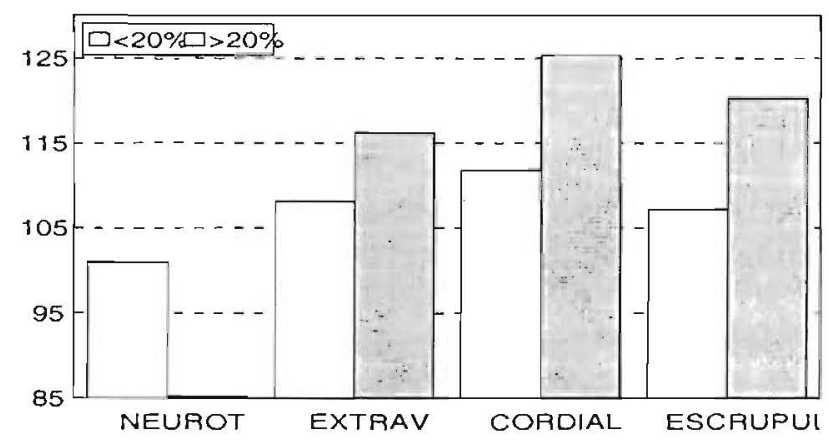

Figura 5. Diferencias estadisticamente significativas de personalidad en grupos extremos (20\%) de astucia/prudencia. La explicación en el texto.

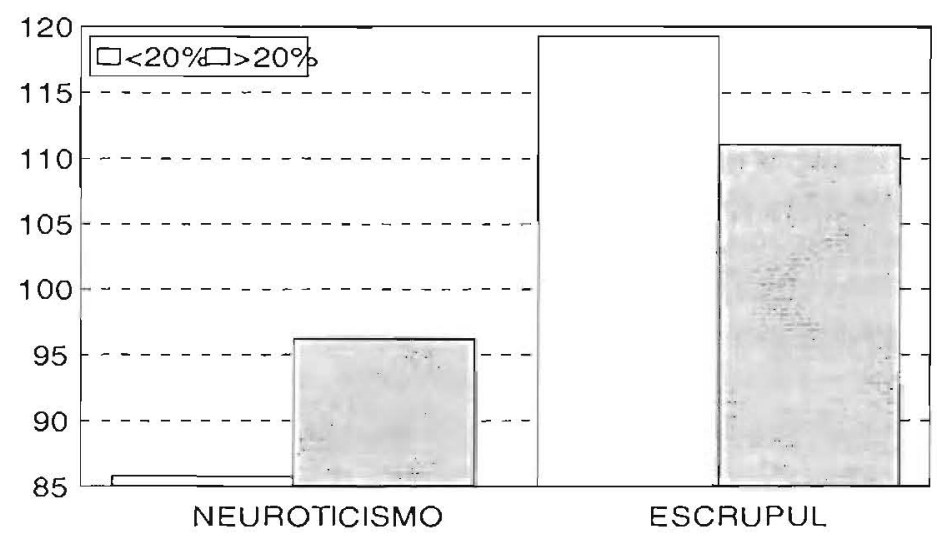

Los resultados confirman que las relaciones entre estos dos grupos de variables (dimensiones de los cinco grandes de personalidad y escalas de sabiduría tradicional) presentan relaciones lineales en la medida en que se obtienen resultados que aunque cualitativamente no parecen ser distintos, sí cualifican los encontrados en el análisis correlacional, que pasaban desapercibidas en aquel análisis:

(a). El factor de estoicismo identificado como constructivismo mental y restricción en placer ofrece relaciones estadísticamente 
significativas y negativas con neuroticismo (a más sabiduría, menos puntuación) y lo contrario sucede con extraversión, cordialidad y escrupulosidad. Ello indicaría que, aunque de manera no muy intensa aunque sí clara, este constructivismo tiende a estar facilitado por puntuaciones altas en extraversión, cordialidad con los demás y escrupulosidad (figura 4).

(b). El primer factor de epicureismo definido como control/ independencia personal con aceptación de jerarquización social está favorecido por una alta puntuación en cordialidad y en escrupulosidad (figura 1).

(c). El segundo factor de epicureismo, definido como inmanencia y devaluación de la muerte tiende a ser favorecido solamente por una puntuación alta en el factor de astucia/prudencia en relaciones interpersonales (figura 2).

(d). El tercer factor de epicureismo, definido como dicotomía placer/ dolor con devaluación del dolor físico está promovido por una puntuación alta en la apertura ala experiencia y una puntuación baja en neuroticismo (figura 3).

(e). Finalmente, el factor del cuestionario de refranes, definido como astucia/prudencia en relaciones personales es promovido por puntuaciones altas en neuroticismo y una puntuación baja en el factor de escrupulosidad (figura 5).

\section{CONCLUSIONES Y DISCUSIÓN}

Los resultados alcanzados en este trabajo permiten concluir que se han contrastado las dos hipótesis racionales que se han formulado al comienzo: (a) que la "sabiduría" se puede entender de diversas maneras y que incluso el "saber de vida" no se resuelve con un único factor funcional sino que posee, al menos tres factores cuando se toman en consideración elementos de tradición popular y filosofías de corte tradicional y (b) que la sabiduría, entendida de esta manera, se presenta como un dominio psicológico algo alejado de la dimensionalización "básica" de la personalidad más utilizada en nuestros días y en nuestra cultura. Asimismo, parece que en un rango de edad muy amplio de juventud y vida adulta, la edad no parece desempeñar un papel muy relevante. 
Por otro lado, parece que pueden rastrearse algunas relaciones entre arribos dominios que, aunque no permiten una predicción clara de un dominio a partir de otro, sj permitirian rastrear con otra lógica estas relaciones. En la medida en que la sabiduría no se presenta en todas las ocasiones y momentos de la vida, es posible que existan un numeroso volumen de factores de personalidad de corte más contextual y que presenten relaciones de mayor intensidad. Nos estamos refiriendo a los factores de nivel intermedio que defiende el modelo de parámetros y que cubren elementos de corte motivacional y de interacción en trabajo, familia y ocio. Estos factores han demostrado su relevancia tanto en el mundo educativo como laboral $y$, como en este mismo número se presenta, asimismo en el dominio de la psicopatología grave (los trastornos de personalidad).

Por otra parte, los resultados sugieren, asimismo, que el peso que ejercen los factores de sabiduría no parece ser el mismo en todo el rango de puntuaciones de personalidad. Es muy posible pensar que la "relevancia" de las dimensiones de personalidad y de sabiduría sean distintas si se posee una puntuación "baja", que si se posee una puntuación "alta" y ello podría ser debido a la existencia de relaciones no lineales y a efectos de "umbral" entre ambos dominios, lo que parece que existe en otros dominios de la psicología (por ejemplo, la última propuesta de Fredrickson y Losada (2005) sobre efectos de la afectividad positiva en el desempeño de tareas muy diversas), tipos de relación que viene defendiendo el autor senior de este trabajo desde hace más de 30 años. Para ello, aparte de ideas hay que aportar resultados y sobre eso esperamos que traten otros trabajos.

\section{REFERENCIAS BIBLIOGRÁFICAS}

Ardelt, M. (2004).- Wisdom as expert knowledge system: A critical review of a contemporary operationalization of an ancient concept, Human Development, 47, 257-285.

Argyle, M. (2001).- The psychology of happiness, $2^{\text {nd }}$ edit., Sussex, Routledge.

Avia, M.D., Sanz, J. \& Sánchez-Bernardos, M.L.(1997).- Versión española del inventario de personalidad NEO revisado NEO-PI-R de Paul T. Costa y Robert R. McCrae (1992), Departamento de 
Psicología Clínica, Universidad Complutense de Madrd, Edición experimental.

Baltes, P. B., Glück, J. \& Kunzmann, U. (2005).- Wisdom: Its structue and function in regulating successful life span. En C. R. Snyder \& S. J. Lopez (Eds.).- Handbook of positive psychology, New York, Oxford University Press, 327-349.

Baltes, P. B. \& Kunzmann, U. (2004).- The two faces of wisdom: Wisdom as a general theory of knowledge and judgment about excellence in mind and virtue vs. wisdom as everyday realization in people and products, Human Development, 47, 290-299.

Costa, P. T. \& McCrae, R. R. (1992).- Revised NEO personality inventory (NEO-PI-R) and NEO five-factor inventory (NEO-FII) professional manual, Odessa, FL, Psychological Assessment Resources.

De Miguel, A. \& Pelechano, V. (2000).- Estructura de personalidad y trastornos de personalidad definidos por clasificación de consenso, Psicologemas, 14, 1-210.

Fredrickson, B. L. (2001).- The role of positive emotions in positive psychology: The broaden-and-build theory of positive emotions, American Psychologist, 56, 218-226.

Fredrickson, B. L. (2005).- Positive emotions, En C. R. Snyder \& S. J. Lopez (eds.).- Handbook of positive psychology, New York, Oxford University Press, 120-134.

Fredrickson, B. L. \& Losada, M. L.(2005).- Positive affect and the complex dynamics of human flourishing, American Psychologist, 60 , 678-686.

Garcia, L. \& Pelechano, V. (2004).- Western traditional wisdom philosophies and personality: a preliminary study, Análisis $y$ Modificación de Conducta, 30, 949-962.

Pelechano, V. (1997).- Una introducción al modelo de parámetros en personalidad. En V. Pelechano (comp.).- Psicología de la personalidad. I. Teorías, Barcelona, Ariel.

Pelechano, V. (2000).- Psicología sistemática de la personalidad, Barcelona, Ariel.

Pelechano, V. (2006).- Sabiduría epicúrea y psicología, Psicothema, en prensa.

Pelechano, V. \& Aguilera, F. J. (2004).- El cuestionario IA de intolerancia de la ambigüedad de Pelechano para adultos: presentación y datos 
de validación interna, convergente y diferencial, Análisis y Modificación de Conducta,30, 627-662.

Pelechano, V., García, L. \& Fumero, A. (2004).- Refranes y sabiduría: el cuestionario REFRUD-1 de Pelechano, Análisis y Modificación de Conducta, 30, 5-50.

Pelechano, V. \& Servando, M. A. (2004).- ¿Qué es la personalidad?, Madrid, Biblioteca Nueva. 